\title{
Separation of the Magnetic Field into External and Internal Parts
}

\author{
N. Olsen · K.-H. Glassmeier $\cdot$ X. Jia
}

Received: 27 March 2009 / Accepted: 2 June 2009 / Published online: 25 July 2009

(C) The Author(s) 2009. This article is published with open access at Springerlink.com

\begin{abstract}
The magnetic field of a planet or a planetary moon contains contributions from a variety of sources in the environment of the body (external sources) and its interior (internal sources). This chapter describes different methods that have been developed for the separation of external and internal source contributions, and their application to selected planets and one of Jupiter's moons, Ganymede.
\end{abstract}

Keywords Spherical harmonic analysis · External current systems · Magnetospheric currents $\cdot$ Earth $\cdot$ Mars $\cdot$ Mercury $\cdot$ Ganymede

\section{Introduction}

Magnetic fields play an important role in physical processes throughout the Universe. In our own solar system the planetary magnetic fields are surprisingly different, even for planets of similar composition. In addition to being evidence of the evolution of the planet the magnetic field exerts a very direct control of the electrodynamic environment.

N. Olsen $(\bowtie)$

DTU Space and Niels Bohr Institute of Copenhagen University, Juliane Maries Vej 30, 2100

Copenhagen $\varnothing$, Denmark

e-mail: nio@space.dtu.dk

K.-H. Glassmeier

Institut für Geophysik und extraterrestrische Physik, TU Braunschweig, Mendelssohnstraße 2-3, 38106

Braunschweig, Germany

K.-H. Glassmeier

Max-Planck-Institut für Sonnensystemforschung, Max-Planck-Strasse 2, 37191 Katlenburg-Lindau, Germany

X. Jia

Department of Earth and Space Sciences and Institute of Geophysics and Planetary Physics, University

of California, Los Angeles, 405 Hilgard Ave., Los Angeles, CA, 90095-1567, USA 
However, the magnetic field measured at or near the surface of a planet is the superposition of contributions from a variety of sources in the planetary environment (e.g. electric currents flowing in the ionosphere and magnetosphere) and its interior (e.g. fluid core, magnetization of rocks in the crust and induced currents in the planetary interior by the timevariations of the external fields). The scientific challenge is the sophisticated separation of these various sources and the accurate determination of the spatial and temporal structure of them.

The first attempt to separate internal and external magnetic field contributions has been performed about 180 years ago by Carl-Friedrich Gauss (1839). He developed the concept of spherical harmonic analysis, applied it to observations of the Earth's magnetic field, and came to the conclusion that by far the largest part of the field originates inside the Earth. The theory of external-internal field separation based on a spherical harmonic expansion will be treated in Sect. 2.1, and application to Earth and Mars (two planets for which globally distributed magnetic field observations obtained from satellites are available) will be discussed in Sects. 3.1 and 3.2.

As an alternative to such a separation by means of spherical harmonic expansion (which does not require a priori knowledge on the spatial structure of the magnetic field since this is estimated from the data), a separation based on physically motivated models of external sources (i.e. knowledge of the time-space structure of the magnetic field and/or the currents generating it) is often more efficient in terms of the required numbers of model parameters to describe the observations. Especially in the case of sparse observations, for instance from a satellite flyby rather than an orbiting satellite, this approach helps to reduce the interpretation ambiguity. An external-internal field separation based on this approach is discussed in Sect. 2.3, and application to Earth and Mercury is treated in Sects. 3.1 and 3.3. Field separation in the presence of plasma interactions, with application to one of Jupiter's moons, Ganymede, is discussed in Sect. 4.

\section{Mathematical Separation of Internal and External Contributions}

\subsection{Separation by Means of Spherical Harmonics and the Theory of Gauss}

In regions without electric currents, and for sufficiently slowly changing fields, the magnetic field $\mathbf{B}=-\nabla V$ can be expressed as the negative gradient of a scalar potential $V$. Because of $\nabla \cdot \mathbf{B}=0$, the potential $V$ has to be a solution of Laplace's equation: $\nabla^{2} V=0$. Under these assumptions the magnetic field is a Laplacian potential field, which puts considerable constraints on the spatial dependency of $\mathbf{B}$.

It is advantageous to use planetocentric spherical coordinates $r, \theta, \phi$ for describing the magnetic field of a planet. Here $r$ is the radius of the point in consideration, $\theta$ is its colatitude, and $\phi$ is its longitude. Assuming that a region of radius $a<r<c$ is free of electric currents (i.e. $\nabla \times \mathbf{B}=0$ everywhere in that shell, cf. Fig. $1 \mathrm{a}), \mathbf{B}=-\nabla V$ in that region, and the potential $V$ can be expanded into series of spherical harmonics.

Solutions of Laplace's equation in spherical harmonics consist of terms with radial dependency $1 / r^{n+1}$ and $r^{n}$, respectively. For $r>a$, only terms $\propto 1 / r^{n+1}$ are physical meaningful solutions and lead to that part, $V^{\text {int }}$, of the potential which describes sources of internal origin (due to electric currents $\mathbf{J}^{\text {int }}$ in the region $r<a$ ). Contrary, in the region $r<c$ only terms $\propto r^{n}$ are physically meaningful; they correspond to the potential part $V^{\text {ext }}$ that describes sources of external origin (due to electric currents $\mathbf{J}^{\text {ext }}$ in the region $r>c$ ). Adding 

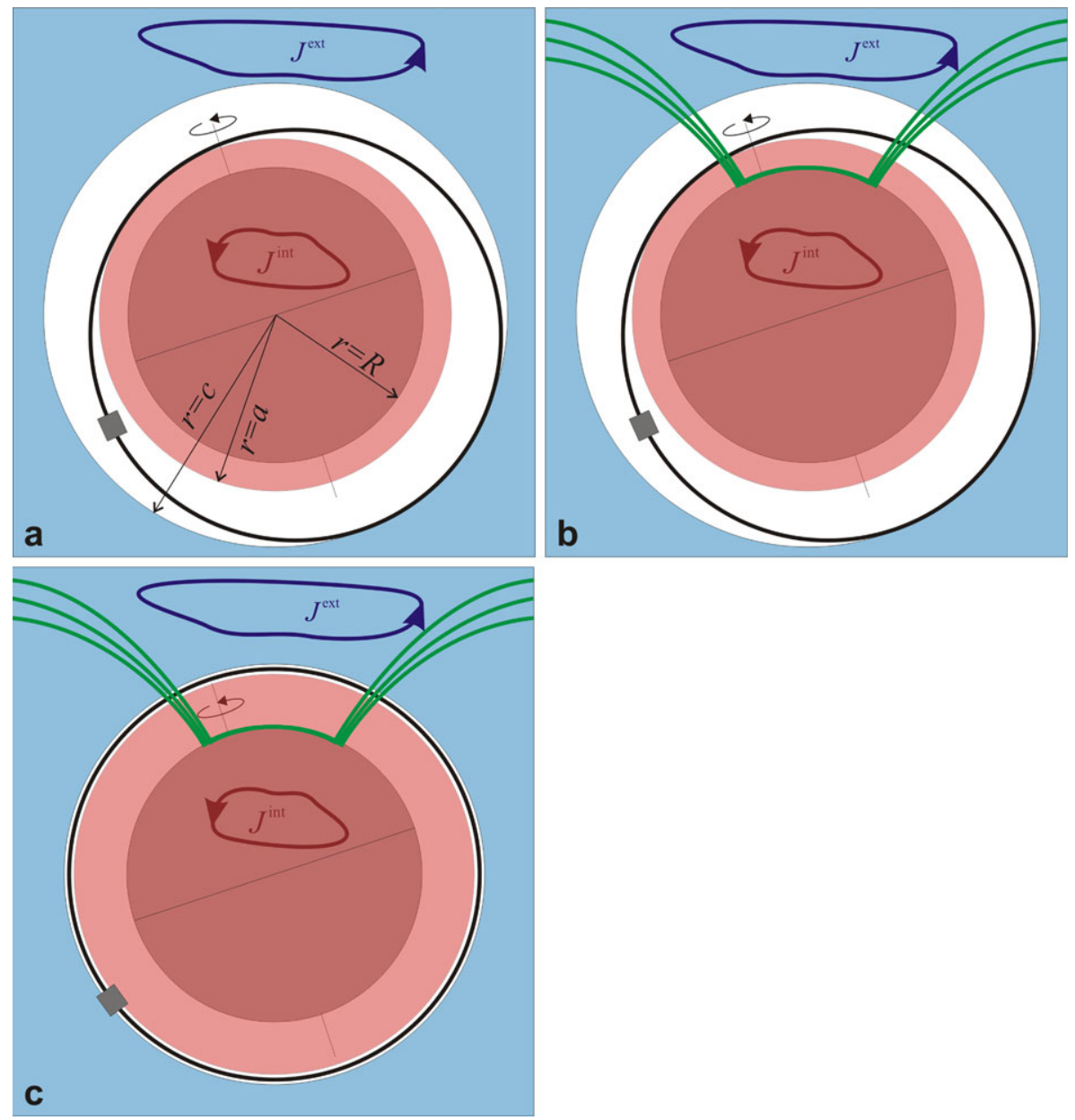

Fig. 1 Scheme of the various source regions. Blue indicates regions that cover the external currents $\mathbf{J}^{\text {ext }}$, red indicates regions that include the internal currents $\mathbf{J}^{\text {int }}$. White regions are free of currents. The black thick circle illustrates the orbit of the satellite (grey box). The planet is indicated by the sphere of radius $R$. Currents that cross satellite altitude are shown in green in panels $\mathbf{b}$ and $\mathbf{c}$. Their existence violates the assumption of a shell $a<r<c$ free of currents shown in panel a

both parts together results in an expansion of $V$ which is valid in the whole current-free region $a<r<c$ :

$$
\begin{aligned}
V= & V^{\mathrm{int}}+V^{\mathrm{ext}} \\
= & R \sum_{n=1}^{N_{\text {int }}} \sum_{m=0}^{n}\left(g_{n}^{m} \cos m \phi+h_{n}^{m} \sin m \phi\right)\left(\frac{R}{r}\right)^{n+1} P_{n}^{m}(\cos \theta) \\
& +R \sum_{n=1}^{N_{\mathrm{ext}}} \sum_{m=0}^{n}\left(q_{n}^{m} \cos m \phi+s_{n}^{m} \sin m \phi\right)\left(\frac{r}{R}\right)^{n} P_{n}^{m}(\cos \theta)
\end{aligned}
$$


(Chapman and Bartels 1940; Langel 1987), where $R$ is a reference radius (typically the mean radius of the planet), $P_{n}^{m}$ are the associated Schmidt semi-normalized Legendre functions, $N_{\text {int }}$ is the maximum degree and order of the internal potential coefficients $g_{n}^{m}, h_{n}^{m}$, and $N_{\text {ext }}$ is that of the external potential coefficients $q_{n}^{m}, s_{n}^{m}$.

In complex notation (and setting for simplicity $N=N_{\text {int }}=N_{\text {ext }}$ as maximum degree and order for both internal and external sources), this equation reads

$$
V=\operatorname{Re}\left\{R \sum_{n=1}^{N} \sum_{m=0}^{n}\left[\iota_{n}^{m}\left(\frac{R}{r}\right)^{n+1}+\epsilon_{n}^{m}\left(\frac{r}{R}\right)^{n}\right] P_{n}^{m} e^{i m \phi}\right\}
$$

where $\iota_{n}^{m}=g_{n}^{m}-i h_{n}^{m}$ and $\epsilon_{n}^{m}=q_{n}^{m}-i s_{n}^{m}$ are the (complex) coefficients describing internal, resp. external, sources, and $\operatorname{Re}\{\cdot\}$ stands for the real part. In following we will drop $\operatorname{Re}\{\cdot\}$ and assume implicitly that only the real part of measured quantities like the magnetic field is taken.

The magnetic field components $\mathbf{B}=\left(B_{r}, B_{\theta}, B_{\phi}\right)^{T}$ (where $B_{r}$ is pointing radially outward, $B_{\theta}$ is pointing Southward, and $B_{\phi}$ is pointing Eastward) follow as

$$
\begin{gathered}
B_{r}=-\frac{\partial V}{\partial r}=\sum_{n, m}\left((n+1) \iota_{n}^{m}\left(\frac{R}{r}\right)^{n+2}-n \epsilon_{n}^{m}\left(\frac{r}{R}\right)^{n-1}\right) P_{n}^{m} e^{i m \phi} \\
B_{\theta}=-\frac{1}{r} \frac{\partial V}{\partial \theta}=\sum_{n, m}\left(\iota_{n}^{m}\left(\frac{R}{r}\right)^{n+2}+\epsilon_{n}^{m}\left(\frac{r}{R}\right)^{n-1}\right) \frac{d P_{n}^{m}}{d \theta} e^{i m \phi} \\
B_{\phi}=-\frac{1}{r \sin \theta} \frac{\partial V}{\partial \phi}=\sum_{n, m}\left(\iota_{n}^{m}\left(\frac{R}{r}\right)^{n+2}+\epsilon_{n}^{m}\left(\frac{r}{R}\right)^{n-1}\right) \frac{i m}{\sin \theta} P_{n}^{m} e^{i m \phi} .
\end{gathered}
$$

A separation of internal and external sources, i.e. a determination of the expansion coefficients $\iota_{n}^{m}$ and $\epsilon_{n}^{m}$, is possible from a combined analysis of radial and horizontal magnetic field components. This becomes obvious when looking at the magnetic field components at the planetary surface $(r=R)$ :

$$
\begin{aligned}
B_{r} & =\sum_{n, m}\left((n+1) \iota_{n}^{m}-n \epsilon_{n}^{m}\right) P_{n}^{m} e^{i m \phi} \\
B_{\theta} & =\sum_{n, m}\left(\iota_{n}^{m}+\epsilon_{n}^{m}\right) \frac{d P_{n}^{m}}{d \theta} e^{i m \phi} \\
B_{\phi} & =\sum_{n, m}\left(\iota_{n}^{m}+\epsilon_{n}^{m}\right) \frac{i m}{\sin \theta} P_{n}^{m} e^{i m \phi}
\end{aligned}
$$

Thus the magnetic horizontal components $B_{\theta}, B_{\phi}$ provide information on the sum of the internal and external field, $\iota_{n}^{m}+\epsilon_{n}^{m}$, while the radial component $B_{r}$ provides information on their difference, $(n+1) \iota_{n}^{m}-n \epsilon_{n}^{m}$. An analysis of all three vector components therefore allows to separate sources of internal and external origin, as first done by Gauss (1839).

Of special interest are the terms with $n=1$; they have a simple geometric meaning. As can be seen from Eq. 3, there is no radial dependency of the magnetic field of external origin for $n=1$, and therefore this part corresponds to a uniform field. If $x, y$ and $z$ define a Cartesian coordinate system with origin in the planetary center, $z$ pointing towards the North pole, $x$ pointing towards the zero meridian $\left(\phi=0^{\circ}\right)$ and $y$ pointing towards the meridian 
$\phi=90^{\circ}$, then the magnetic field component in $z$ direction is given by $q_{1}^{0}=\operatorname{Re}\left\{\epsilon_{1}^{0}\right\}$, while those in the directions of $x$ and $y$ are given by $q_{1}^{1}=\operatorname{Re}\left\{\epsilon_{1}^{1}\right\}$ and $s_{1}^{1}=-\operatorname{Im}\left\{\epsilon_{1}^{1}\right\}$, respectively. For the internal part, $g_{1}^{0}=\operatorname{Re}\left\{\iota_{1}^{0}\right\}$ represents a dipole at the planets center that is aligned with $z$, while $g_{1}^{1}=\operatorname{Re}\left\{\iota_{1}^{1}\right\}$ and $h_{1}^{1}=-\operatorname{Im}\left\{\iota_{1}^{1}\right\}$ are dipoles in the equatorial plane pointing towards $x$, resp. $y$.

It is important to notice that the above described separation into internal and external sources is done with respect to the altitude of the observations, not with respect to the planetary surface. As a consequence, sources between surface and satellite altitude (e.g. ionospheric currents) are seen as internal fields by satellites, although they are external to the planet. Contributions internal to the planet may originate from dynamo action in planetary cores ("core field"), from magnetized material, typically near the surface ("crustal field"), and from electromagnetically induced currents in the conducting planetary interior ("induced fields", cf. Saur et al. 2009).

A representation of the magnetic field by means of a spherical harmonic expansion of the scalar potential, as given by Eqs. 1-4, is only possible if the whole region in which the data are acquired is free of electric currents. In practice this is often not the case when satellite data are analyzed, since electric currents in regions sampled by the satellite violate this condition (cf. the high-latitude field-aligned currents shown in Fig. 1b). Even if most of the region (for instance the non-polar region) is free of electric currents (i.e. $\nabla \times \mathbf{B}=0$ ), the existence of currents at some latitudes violates the assumption that the whole region has to be source free, and therefore a spherical harmonic representation of the magnetic field is not possible. (Note that a local representation of the magnetic field $\mathbf{B}=-\nabla V$ in the current free regions is still achievable although the global expansion of $V$ into series of spherical harmonics is not.)

\subsection{Separation in Current-Carrying Regions}

In regions with electric currents the magnetic field $\mathbf{B}$ cannot be expressed as the negative gradient of a scalar potential only, but is defined by the expression $\mathbf{B}=-\nabla V+\nabla \times \mathbf{A}$, where $\mathbf{A}$ is a vector potential. In this case the Gauss algorithm outlined above cannot be used and a separation of the measured field into its various contributions is more difficult. Assuming that a region of radius $a<r<c$ is filled with electric currents a representation of the magnetic field in this shell by means of poloidal and toroidal vector field $\mathbf{B}^{\text {pol }}$ and $\mathbf{B}^{\text {tor }}$ is useful:

$$
\mathbf{B}=\mathbf{B}^{\text {pol }}+\mathbf{B}^{\text {tor }}
$$

where

$$
\begin{aligned}
& \mathbf{B}^{\text {pol }}=\nabla \times(\mathbf{r} \times \nabla P) \\
& \mathbf{B}^{\text {tor }}=\mathbf{r} \times \nabla T .
\end{aligned}
$$

The two scalar fields $P$ and $T$ are called the poloidal and toroidal scalars of $\mathbf{B}$. Representing a magnetic vector field by its poloidal and toroidal vector fields is called the Mie representation of B. It has extensively been discussed by Backus (1986) and is formally not restricted to spherical shells and volume regions between spherical shells, but is also applicable on more general surfaces and volumes generated by two such surfaces.

Consider magnetic field measurements taken on a spherical shell, either a planetary surface or the shell described by a polar orbiting spacecraft. Following Backus (1986), the observed magnetic field $\mathbf{B}$ can be decomposed into a poloidal part of internal origin (sources 
internal to the observation altitude), $\mathbf{B}^{\text {pol,int }}$, a poloidal part of external origin, $\mathbf{B}^{\text {pol,ext }}$, and a third (toroidal) part, $\mathbf{B}^{\text {tor }}$. This toroidal part is due to radial currents at the shell. If $\mathbf{B}$ is known everywhere on the shell, then also the three contributions can be found everywhere on the shell. The internal and external poloidal vector fields $\mathbf{B}^{\text {pol,int }}$ and $\mathbf{B}^{\text {pol,ext }}$ can be determined, much as the Gauss algorithm can be used to separate internal and external contributions in case of a current-free situation. However, it should be noted that the internal contribution is due to all the electric currents flowing inside the sphere. Nothing can be said about the currents in the dynamo region, where the planetary field is generated. All the currents flowing inside the sphere add up in a poloidal contribution at the surface of the sphere.

If there are currents in a thick shell formed by e.g. spheres with the radius of the apoapsis and the periapsis of a spacecraft orbiting a planet (cf. Fig. 1b) a Mie representation can still be used. In this case the scalar fields $P$ and $T$ are functions in the shell uniquely determined by the magnetic field $\mathbf{B}$ in the shell. An expansion of $P$ and $T$ into spherical harmonics is possible, but requires expansion coefficients which depend on the radial distance $r$ within the shell. Without further constrains determination of the expansion coefficients requires knowledge of $\mathbf{B}$ everywhere in the shell. An a priori physical model, based on e.g. simulation results of the interaction of the solar wind with the planet and/or its internal magnetic field, could provide such constrains.

However, if the data are acquired in a thin shell (of thickness $c-a \ll b=(a+c) / 2$, cf. Fig. 1c), an extension of the classical spherical harmonic representation as developed by Gauss is possible. As pointed out by Backus (1986), the observed magnetic field B can be decomposed into a part of internal origin (sources internal to the observation altitude), $\mathbf{B}^{\text {int }}=-\nabla V^{\text {int }}$, a part of external origin, $\mathbf{B}^{\text {ext }}=-\nabla V^{\text {ext }}$, and the third (toroidal) part, $\mathbf{B}^{\text {tor }}$, that is due to in-situ (radial) currents at satellite altitude:

$$
\begin{aligned}
\mathbf{B}= & \mathbf{B}^{\mathrm{int}}+\mathbf{B}^{\mathrm{ext}}+\mathbf{B}^{\mathrm{tor}} \\
= & -\nabla\left[R \sum_{n, m}\left(\frac{R}{r}\right)^{n+1} \iota_{n}^{m} P_{n}^{m} e^{i m \phi}\right] \\
& -\nabla\left[R \sum_{n, m}\left(\frac{r}{R}\right)^{n} \varepsilon_{n}^{m} P_{n}^{m} e^{i m \phi}\right] \\
& -\nabla \times\left[\mathbf{r} \sum_{n, m}\left(\frac{R}{r}\right) \psi_{n}^{m} P_{n}^{m} e^{i m \phi}\right] .
\end{aligned}
$$

Once the expansion coefficients $\phi_{n}^{m}$ are known, the radial current density through the shell $r=b$ can be found from

$$
J_{r}(b, \theta, \phi)=\frac{1}{\mu_{0} b} \sum_{n, m} n(n+1)\left(\frac{R}{b}\right) \psi_{n}^{m} P_{n}^{m} e^{i m \phi} .
$$

The mean-square value (over the sphere with radius $r$ ) of the magnetic field intensity produced by spherical harmonics of degree $n$ has been introduced independently by Mauersberger (1956) and Lowes (1966) and is denoted by $W_{n}$. For a magnetic field containing internal, external and toroidal parts, $W_{n}$ is given by Backus (1986), Eq. 81

$$
\begin{aligned}
W_{n}(r) & =W_{n}^{\mathrm{int}}(r)+W_{n}^{\mathrm{ext}}(r)+W_{n}^{\mathrm{tor}}(r) \\
& =(n+1)\left(\frac{r}{R}\right)^{2 n+4} \sum_{m=0}^{n}\left|\iota_{n}^{m}\right|^{2}
\end{aligned}
$$




$$
\begin{aligned}
& +n\left(\frac{R}{r}\right)^{2 n-2} \sum_{m=0}^{n}\left|\epsilon_{n}^{m}\right|^{2} \\
& +\frac{n(n+1)}{2 n+1}\left(\frac{r}{R}\right)^{2} \sum_{m=0}^{n}\left|\psi_{n}^{m}\right|^{2} .
\end{aligned}
$$

At the planetary surface, $r=R$, this equation reduces to

$$
\begin{aligned}
W_{n}= & (n+1) \sum_{m=0}^{n}\left|\iota_{n}^{m}\right|^{2} \\
& +n \sum_{m=0}^{n}\left|\epsilon_{n}^{m}\right|^{2} \\
& +\frac{n(n+1)}{2 n+1} \sum_{m=0}^{n}\left|\psi_{n}^{m}\right|^{2} .
\end{aligned}
$$

Olsen (1997) made extensive use of the above described approach when studying $F$ region currents in the terrestrial ionosphere. Analysing vector magnetic field observations taken by the Magsat satellite in 1979 and 1980, he determined the toroidal expansion coefficients $\psi_{n}^{m}$ separately for morning and evening in local time, and for two months of data centered at December 21, 1979, and March 21, 1980, respectively. Global maps of the radial current density $J_{r}$ were then synthesized using Eq. 8. These maps show the well-known field-aligned currents at polar latitudes (average current density $J_{r} \lesssim 200 \mathrm{nA} / \mathrm{m}^{2}$ ) and the meridional current system of the terrestrial equatorial electrojet $\left(J_{r} \approx 10-20 \mathrm{nA} / \mathrm{m}^{2}\right)$ during dusk. In addition, these maps revealed for the first time a midlatitude interhemispheric current system in the terrestrial ionosphere, with current direction from the winter to the summer hemisphere during dusk and in opposite direction during dawn. This is in agreement with predictions by three-dimensional models of the ionospheric dynamo.

An application of the poloidal-toroidal decomposition approach to Mars is discussed in Sect. 3.2.

\subsection{Internal-External Field Separation Based on Models of External Source Fields}

If magnetic field measurements are made in current-carrying regions a detailed knowledge of the electric current distribution is necessary for a final separation of the measured field into its internal and external parts. Such a knowledge requires, for example, a larger number of spacecraft onboard which magnetic field measurements are made. Currently only the four Cluster spacecraft, orbiting round Earth, provide such a possibility to a limited extend (Dunlop et al. 2002). (As a near-Earth equivalent, the two side-by-side flying satellites of the Swarm constellation mission (Friis-Christensen et al. 2006) to be launched in 2011 will allow for an instantaneous determination of the radial current density in the terrestrial $F$ region ionosphere.)

However, in the terrestrial magnetosphere the distribution of electric currents is not uniform, but largely localized in the magnetopause and ring current regions as well as in the ionosphere. Therefore, most of the measurements are done in almost current-free regions, where the Gauss algorithm can be used and the description of the magnetic field by a scalar magnetic potential is justified. However, it is practise not to determine the external field directly from the actual data, but to make extensive use of parameterized external magnetic field models. 
There are three categories of knowledge on external field geometry that can be used in this situation to achieve an external-internal field separation:

- choice of appropriate coordinate systems;

- parameterized models of ionospheric sources (Lühr et al. 2009);

- parameterized models of magnetospheric sources, for instance the Tsyganenko model series (e.g., Tsyganenko 1989, 1995, 2002a, 2002b; Tsyganenko and Sitnov 2007).

Concerning the first category, it is useful to remember that internal sources are often fixed with respect to the planet (magnetic fields due to induced currents in the planetary interior are an exception) and thus follow its rotation, while many external fields are often fixed with respect to the sun. Describing external fields in a coordinate frame that follows the (apparent) movement of the sun is therefore advantageous. On planets with Earth-like magnetospheres the use of Solar Magnetospheric (SM) coordinates for describing near magnetospheric currents like the ring-current, and of Geocentric Solar Magnetospheric (GSM) coordinates for describing far magnetospheric current systems like the tail currents has been turned out to be useful (Maus and Lühr 2005; Olsen et al. 2006). A definition of the SM and GSM coordinate frames can be found in Kivelson and Russell (1995).

Concerning the second category, extensive modelling of the ionospheric current systems and their magnetic fields have been done. For a recent study and review reference is made to Lühr et al. (2009) and references therein. Such modelling is indispensable for a determination of the spherical harmonic expansion coefficients of the geomagnetic field up to very large degree.

The third category, magnetospheric magnetic field modelling, uses parameterized field models based on theoretical models of the magnetosphere. The model parameter are determined from actually measured field values. The models suggested by Tsyganenko and co-workers (Tsyganenko 1989, 1995, 2002a, 2002b; Tsyganenko and Sitnov 2007) are the most widely used models in this respect. In these models the external magnetic field of magnetospheric origin, $\mathbf{B}^{\text {ext }}$, is described as the sum

$$
\mathbf{B}^{\mathrm{ext}}=\mathbf{B}^{\mathrm{CF}}+\mathbf{B}^{\mathrm{RC}}+\mathbf{B}^{\mathrm{T}}+\mathbf{B}^{\mathrm{FAC}}+\mathbf{B}^{\mathrm{OF}},
$$

where $\mathbf{B}^{\mathrm{CF}}, \mathbf{B}^{\mathrm{RC}}, \mathbf{B}^{\mathrm{T}}$, and $\mathbf{B}^{\mathrm{FAC}}$ denote field contributions from the Chapman-Ferraro currents flowing in the magnetopause, the magnetospheric ring current, magnetotail currents, and field-aligned currents, respectively. The contribution $\mathbf{B}^{\mathrm{OF}}$ denotes any other field contribution one would like to incorporate into the model. For the various contributions analytical descriptions are derived with parameters depending on solar wind dynamic pressure, the interplanetary magnetic field, or magnetospheric activity as described by the $\mathrm{D}_{s t}$ or $K p$ indices.

The magnetic field $\mathbf{B}^{\mathrm{CF}}$ of the Chapman-Ferraro currents is described as the gradient of a scalar potential

$$
\mathbf{B}^{\mathrm{CF}}=-\nabla V^{\mathrm{CF}}
$$

where the scalar potential $V^{\mathrm{CF}}$ can be derived by solving the Neumann problem

$$
\begin{aligned}
\nabla^{2} V^{\mathrm{CF}} & =0 \\
\left.\frac{\partial V^{\mathrm{CF}}}{\partial n}\right|_{S}-B_{n}^{\mathrm{int}} & =0 .
\end{aligned}
$$


Here the subscript $n$ denotes the component normal to the magnetopause surface $S$. For this surface $S$ a suitable description as an axially symmetric ellipsoid or paraboloid needs to be found. The Neumann condition also implies that the normal component of the magnetic field at the boundary vanishes. This corresponds to a so-called closed magnetosphere. Other conditions are possible, depending on the physical system to be modelled.

For the scalar potential $V^{\mathrm{CF}}$ a suitable analytical representation is necessary. A flexible choice is a representation (Tsyganenko 1995)

$$
V^{\mathrm{CF}}=\sum_{i, k} a_{i} \chi\left(b_{k}, \mathbf{r}\right)
$$

where $a_{i}$ are the expansion coefficients and $\chi\left(b_{k}, \mathbf{r}\right)$ can be any harmonic function of the position vector $\mathbf{r}$ and some parameter $b_{k}$.

The magnetic field contribution of the cross-tail current, for example, can be described by a vector potential $\mathbf{A}=A_{\phi} \hat{\mathbf{e}}_{\phi}$ with a suitable parameterized description of the azimuthal component $A_{\phi}$ of the vector potential; usually cylindrical coordinates are used. In similar ways the further contributions can be represented.

These parameterized models are to a certain extend comparable to the spherical harmonic expansion introduced by Carl-Friedrich Gauss as in both descriptions the entire field is decomposed into its various contributions. However, the Gauss description uses a set of complete orthogonal functions, spherical harmonics, to describe the field. This description is a purely mathematical, not requiring any physical argument for its usefulness. A description as used by Tsyganenko and others relies on physical insight into the problem, as knowledge about the form of the magnetopause, its current distribution, or the spatial structure of the ring current distribution is required. This a priori, physical input to the model allows to reduce the number of independent parameters. But, in a mathematical sense the description is not complete. Nevertheless, parameterized models are very successful and allow already a very good description of the external field contribution.

\section{Application to Selected Planets}

\subsection{Earth}

After the pioneering work of Gauss (1839), attempts to separate external and internal contributions have been performed by Schmidt (1895, 1898), Fritsche (1900) and Bauer (1923). These early determinations found external field contributions of the order of few hundred nT, which is far too high for average geomagnetic activity conditions. The large values are due to spatial aliasing caused by the very uneven distribution of the ground data that have been used in these investigations. Only satellites are able to provide the uniform data coverage that is necessary for a reliable external-internal field separation. Although magnetic intensity data taken by the POGO satellites improved the situation considerably, it was not before the availability of vector satellite observations that a reliable determination of the large-scale magnetospheric field of about $20 \mathrm{nT}$ (Langel and Estes 1985a, 1985b) during geomagnetic quiet times was possible. More recent satellite data confirmed this value (Maus et al. 2005; Olsen et al. 2006), and furthermore demonstrated that about half of it is due to near magnetospheric sources (best described in SM coordinates) while the other half is due to more distant sources (and follow the GSM frame).

The most complete external-internal separation of the Earth's magnetic field is given by the Comprehensive Model by Sabaka and co-workers. In its present version called CM4 


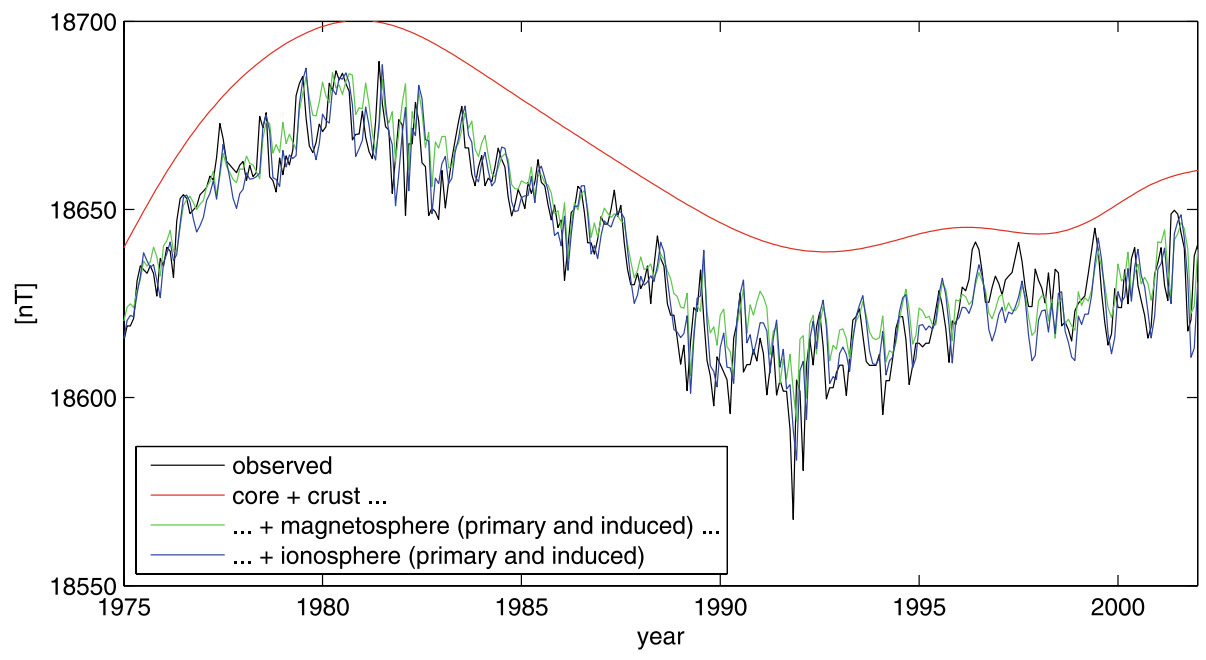

Fig. 2 Observed and predicted (from CM4) monthly mean values of the $X$ component at Niemegk/Germany

(Sabaka et al. 2004), the model attempts to describe the various components of the nearEarth magnetic field by co-estimating the internal, magnetospheric and ionospheric fields (with their secondary, Earth-induced counterparts), in one huge inversion process.

By a joint analysis of ground-based and satellite data it was possible to separate Earthinternal sources, ionospheric sources (which are external to ground, but internal to satellites) and magnetospheric sources (external to ground and low-orbiting satellites).

As an example for the ability of CM4 to separate the various sources, Fig. 2 shows observed monthly mean values of the $X$ (North) component at the Niemegk observatory in Germany (black line) together with CM4 predictions. The red curve shows the internal (core and crustal) part; adding the magnetospheric field (including its induced counterpart) results in the green curve, and adding the ionospheric (plus induced) contribution leads finally to the blue curve. Internal and external field contributions together (blue curve) nicely fits the observed field (black curve).

The comprehensive approach has recently been applied to synthetic satellite data (Sabaka and Olsen 2006), which confirmed that a proper separation of the various source contributions is possible.

\subsection{Mars}

Models of the Martian magnetic field usually describe only internal sources, given by the first part of Eq. 7. However, the simultaneous estimation of internal, external and toroidal magnetic field contributions has turned out to be an advantage for studying the terrestrial magnetic field (Sabaka et al. 2004), and an application to Mars, a planet for which magnetic data from the mapping-quality satellite Mars Global Surveyor (MGS) are available, is straightforward.

To demonstrate this, we used MGS mapping data (taken at about $400 \mathrm{~km}$ altitude) and performed a separate analysis of dayside and nightside data, respectively. Data were sampled between March and November 1999, which yields about 50000 vector triplets in each data set. Following Eq. 7, we solved for internal (up to degree/order $n=40$ ), external (up to $n=20$ ), and toroidal (up to $n=20$ ) field contributions. The external current systems are 
expected to be highly time variable, but here we only consider the time-independent part of the field.

The radial magnetic field component at $400 \mathrm{~km}$ altitude due to external, respectively internal, sources as estimated from night-side data only are shown in Figs. 3a and 3b. The internal field is concentrated in the southern highlands where it is about 10 times stronger than the external field, which, however, is of approximately similar strength everywhere but especially dominant at polar latitudes. Figure $3 \mathrm{c}$ shows the external field obtained from the day-side data; the lower right panel presents the difference between the external field determined from day-side data minus that derived from night-side data. Note that "internal" refers to sources below satellite altitude $(400 \mathrm{~km})$ and includes contributions from ionospheric currents. This is confirmed by the fact that the additional "internal" field on the day-side is about twice as strong as the corresponding "external" field, which is not possible from purely electromagnetic induction in the Martian interior.

Figure 4 shows magnetic power-spectra of the various contributions (internal, external and toroidal) to the Martian magnetic field at surface (left) and $400 \mathrm{~km}$ altitude (right), determined from day-side and night-side observations, respectively. Also shown is the spectrum of the model of Cain et al. (2003). At satellite altitude, contributions from in-situ currents (toroidal magnetic field) exceed those from external sources, even during night-time.

\subsection{Mercury}

Currently the planetary magnetic field of Mercury is not well known. The planet was first visited by the Mariner 10 spacecraft in 1974. The discovery of a magnetic field was certainly a major surprise (Ness et al. 1974). Recently, Mercury was visited again by the MESSENGER spacecraft and additional magnetic field measurements have been possible (Anderson et al. 2008). Due to the limited number of flybys determination of the planetary magnetic field is still in its infancy. This will only change when the MESSENGER spacecraft will start to orbit planet Mercury in 2011 and once the European-Japanese dual spacecraft mission BepiColombo with its two spacecraft Mercury Planetary Orbiter (MPO) and Mercury Magnetospheric Orbiter (MMO), both equipped with magnetometer systems (Glassmeier et al. 2009; Baumjohann et al. 2009), are inserted into polar orbits around Mercury in 2019.

The dipole moments estimated up to now using different approaches significantly differ (the minus sign indicates southward moment): $-170 \mathrm{nT} R_{\mathrm{M}}^{3}$ (Jackson and Beard 1977; Whang 1977), $-192 \mathrm{nT} R_{\mathrm{M}}^{3}$ (Alexeev et al. 2008), $-287 \mathrm{nT} R_{\mathrm{M}}^{3}$ (Korth et al. 2004), -(230 - 290) nT $R_{\mathrm{M}}^{3}$ (Anderson et al. 2008). The most recent joint analysis of Mariner 10 and Messenger observations by Uno et al. (2009) suggest a dipole moment $-227 \mathrm{nT} R_{\mathrm{M}}^{3}$. Higher order moments are to a large extend unconstrained.

The differences mainly result from insufficient data coverage, but also indicate incomplete separation of internal and external contributions by the different magnetospheric magnetic field models used. Knowledge of the external fields is certainly a primary limiting factor in extracting reliable information from the data on Mercury's internal magnetic field.

First models of the Hermean magnetospheric field were provided by e.g. Jackson and Beard (1977) and Whang (1977) with significant recent improvements reached by Giampieri and Balogh (2001), Korth et al. (2004), Grosser et al. (2004), Scuffham and Balogh (2006), and Alexeev et al. (2008). The model used by Jackson and Beard (1977) is a scaled version of the terrestrial magnetic field model of Choe and Bearda (1974a, 1974b). The subsolar magnetic field magnitude ratio is used as a scaling parameter.

The field model of Whang (1977) uses an image dipole to describe the magnetic effect of magnetopause currents and a finite-thickness tail current sheet to model the tail magnetic 

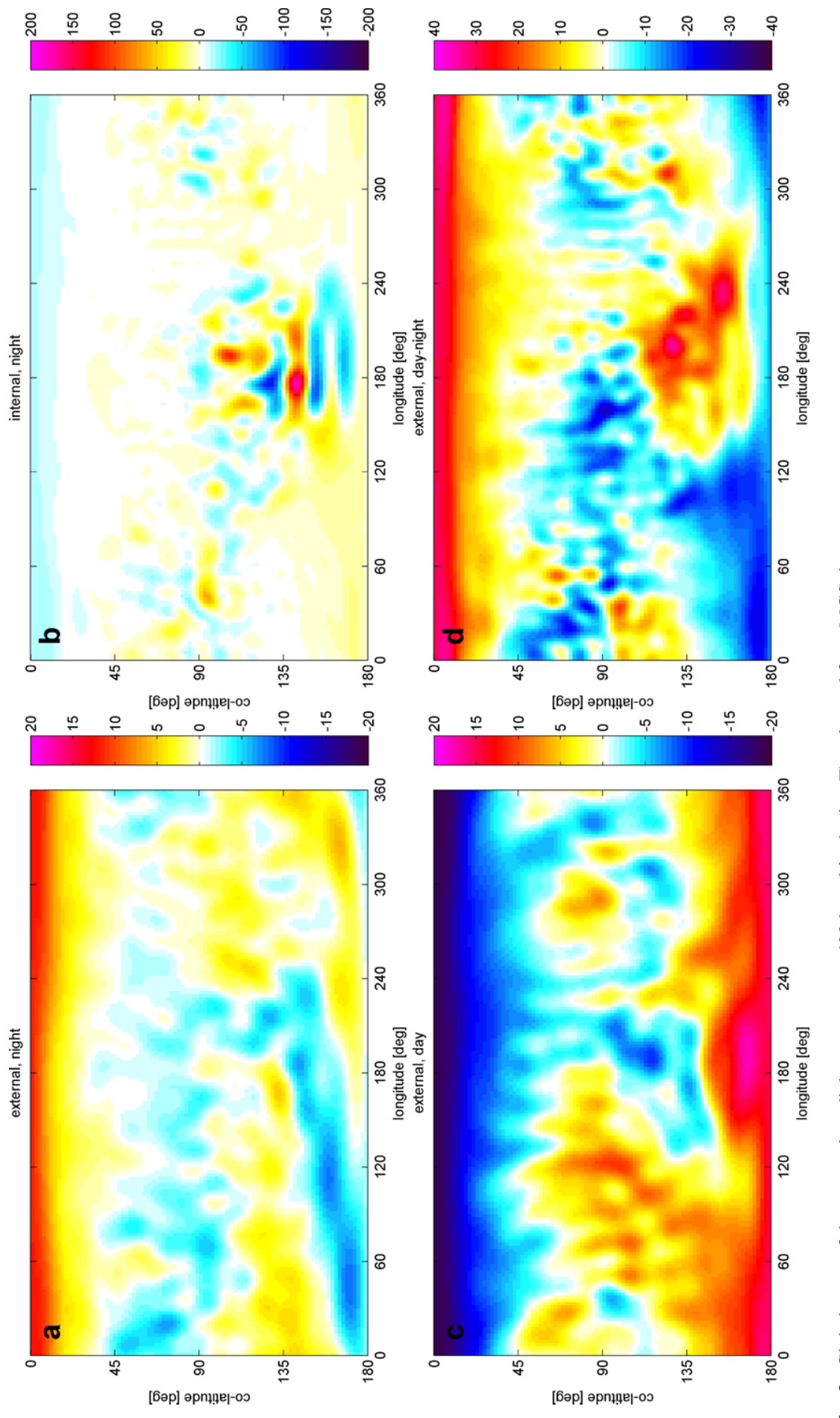

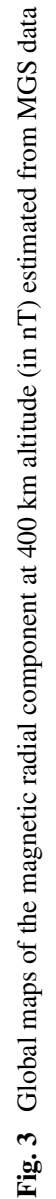



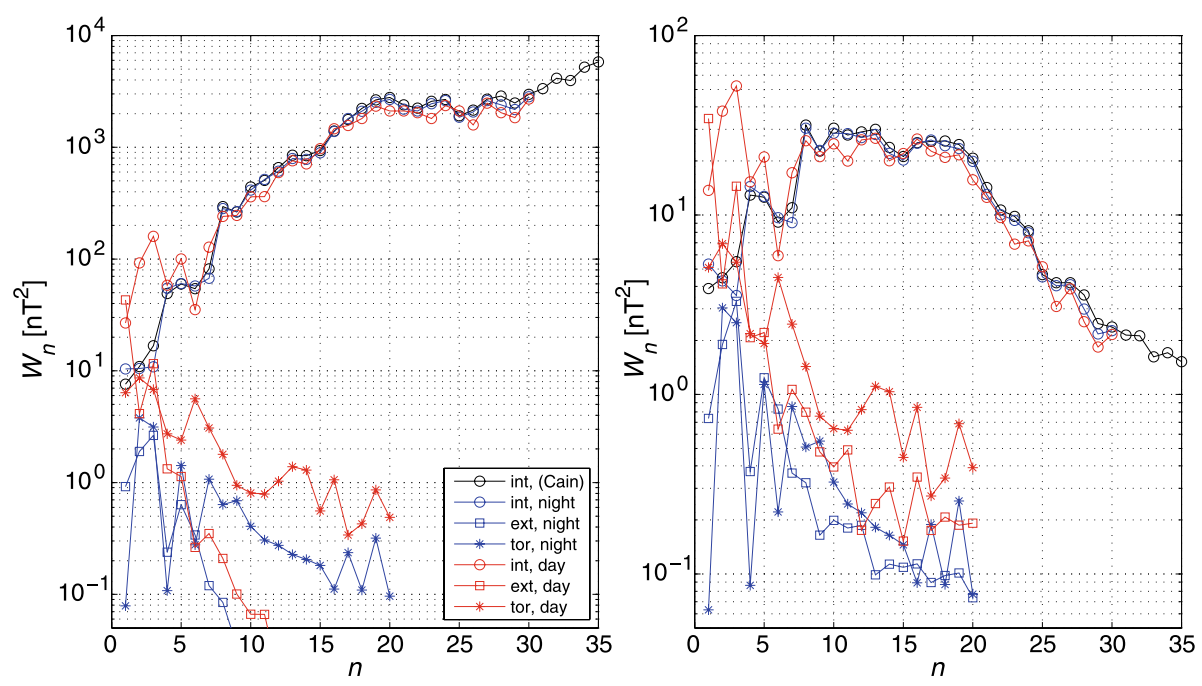

Fig. 4 Power spectra of internal, external and toroidal contributions to the Martian magnetic field at surface (left) and $400 \mathrm{~km}$ altitude (right)

field. Korth et al. (2004) use a scaled version of the Tsyganenko-96 model (Tsyganenko 1995) with any ring current contribution neglected. A somewhat different approach have Alexeev et al. (2008), who use a magnetopause which is a paraboloid of rotation around the Sun-planet line. Magnetic fields due to electric currents on this magnetopause and the tail current system are obtained by appropriate shielding fields to confine the various fields of magnetospheric origin inside the region defined by the magnetopause.

All these models assume sheet current distributions generating the magnetospheric magnetic field. Therefore a scalar potential representation as in Eq. 1 is possible in most regions of the magnetosphere. However, this is the limiting factor of the field models used so far as the weak planetary magnetic field causes a rather small magnetosphere with the planetocentric stand-off distance of the magnetopause at $R_{\mathrm{MP}} \approx 1.7 R_{\mathrm{M}}$ (where $R_{\mathrm{M}}=2440 \mathrm{~km}$ is the Hermean planetary radius) (Siscoe and Christopher 1975). The magnetic field of the Chapman-Ferraro currents flowing in this close-to-the-planet magnetopause causes a surface magnetic field of the order of $100 \mathrm{nT}$ (e.g. Grosser et al. 2004). As the thickness of the Hermean magnetopause is of the order of a few hundred kilometers (e.g. Russell and Walker 1985) this implies that the magnetopause current layer at Mercury is not just a current sheet, but a broad current-carrying layer.

The complexity and yet unexplored nature of the Hermean magnetopause is also demonstrated by the recent Messenger observations (Slavin et al. 2008), where indications for a double-magnetopause type structure were identified. This poses another major problem for the separation of internal and external field contributions as the regions where magnetic field measurements are taken is not necessarily current-free. Thus, the Gauss algorithm is strictly not applicable in most of the magnetospheric regions at Mercury. Also the current parameterized models fail in describing this new situation. The more general Mie representation (see Eq. 5) and related separation algorithms are a more appropriate tools to describe and analyze the available observations.

Incorrectly assuming a current-free shell in which data are taken and application of the Gauss algorithm has major effects on the estimated spherical harmonic expansion coefficients. Using a spherically shaped magnetosphere and azimuthally flowing magnetopause 
Fig. 5 Comparison of the estimated dipole field coefficient with respect to the true internal value. A spherical magnetosphere with a finite magnetopause and azimuthally flowing electric currents is assumed. The estimate is based on erroneously assuming a scalar potential field description of the magnetic field

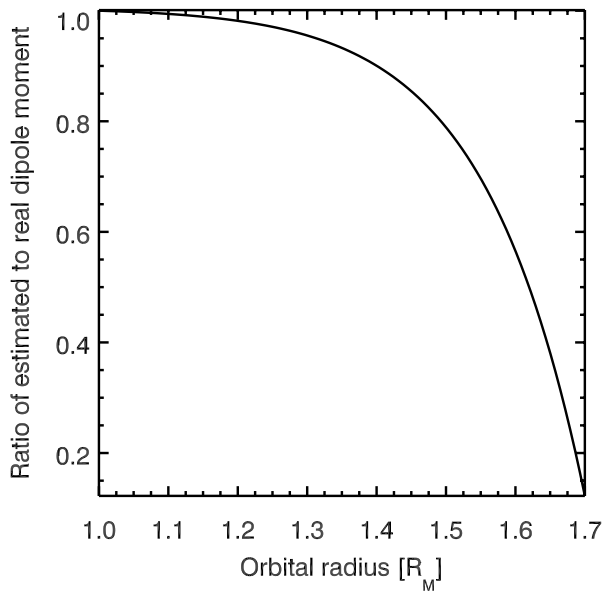

current density with a Rayleigh distribution-type variation in radial direction Heyner (2007) and Glassmeier et al. (2009) modelled external magnetic fields to which an internal dipole field was added. The current distribution decays towards the planet with a scale of $500 \mathrm{~km}$ and towards the magnetosheath with $50 \mathrm{~km}$; maximum current density reaches $2.8 \times 10^{-7} \mathrm{Am}^{2}$. The magnetopause is located at $1.7 R_{\mathrm{M}}$.

Figure 5 displays the results of an inversion of magnetic field observations taken along circular polar orbits around the model planet at various radii. The closer the spacecraft is flying to the current-carrying magnetopause the less reliable is the estimated dipole moment. Magnetic fields of the inner current layers actually shield the internal dipole. In the terrestrial magnetosphere one can avoid this by not taking into account measurements made close to the magnetopause. However, at Mercury this possibility does not exists. For example, the MPO spacecraft of the BepiColombo mission will take magnetic field measurements in a polar orbit around Mercury with a periherm of $1.16 R_{\mathrm{M}}$ and an apoherm of $1.6 R_{\mathrm{M}}$. MPO will fly at a relative distance to the magnetopause between $0.68-0.94 R_{\mathrm{MP}}$. In the terrestrial magnetosphere this would correspond to an orbit with a perigee of about $43000 \mathrm{~km}$ and an apogee of about $59000 \mathrm{~km}$.

Due to the sparsity of available data the problem of a proper separation of the fields of internal and external origin has not yet been solved. New tools need to be developed for an efficient exploration of the future magnetic field data from the Messenger and BepiColombo magnetometer experiments (Anderson et al. 2007; Baumjohann et al. 2009; Glassmeier et al. 2009). In a first step, one needs to estimate the strength of any local electric current flows. The BepiColombo spacecraft will provide dual-point magnetic field observations. Around periherm passage MPO and MMO will be separated by a distance of $50-100 \mathrm{~km}$ in radial direction. This allows for a determination of the radial gradient. Neglecting displacement currents we have $\nabla \times \mathbf{B}=\mu_{0} \mathbf{J}$, which reads in spherical coordinates close to the equator $\left(\theta \approx 90^{\circ}\right)$ :

$$
\begin{aligned}
& \mu_{0} J_{r}=\frac{1}{r}\left(\frac{\partial B_{\phi}}{\partial \theta}-\frac{\partial B_{\theta}}{\partial \phi}\right), \\
& \mu_{0} J_{\theta}=\frac{1}{r}\left(\frac{\partial B_{r}}{\partial \phi}-\frac{\partial r B_{\phi}}{\partial r}\right),
\end{aligned}
$$




$$
\mu_{0} J_{\phi}=\frac{1}{r}\left(\frac{\partial r B_{\theta}}{\partial r}-\frac{\partial B_{r}}{\partial \theta}\right)
$$

To first order it is justified to neglect the azimuthal and field-aligned variations $\partial / \partial \phi$ and $\partial / \partial \theta$ of the magnetic field. Thus, MPO/MMO two-point magnetic field measurements will provide a first-order estimate of azimuthal and field-aligned current flows in the magnetosphere of Mercury. If these currents are large, the Gauss algorithm and any similar approach can not be used.

\section{External-Internal Field Separation in the Presence of Plasma Interactions}

The interaction between a flowing plasma and a magnetized obstacle generates currents that cause the magnetic field in the vicinity of the obstacle to deviate significantly from the superposition of the internal field and the background field. Description of the external field perturbations caused by plasma interactions can be provided by physically motivated models of the global interaction. For instance, in an attempt to improve the constraints on the inductive response of Europa, one of the Jovian moons that generates an induced magnetic field through electromagnetic induction, Schilling et al. (2007) applied a global MHD model to account for the effects arising from plasma interactions. Here we use Ganymede, another Galilean satellite of Jupiter, as an example to discuss some issues related to the separation of an internal field from the field arising from external sources including the perturbations introduced by plasma interactions.

Ganymede, the largest moon in the solar system, has an intrinsic magnetic field that is sufficiently strong to stand off Jupiter's flowing magnetospheric plasma above its surface and form a magnetosphere (Kivelson et al. 1996). Similar to that of the planet Mercury, Ganymede's magnetosphere is rather small with the typical stand-off distance of the magnetopause $R_{\mathrm{MP}} \approx 1.8 R_{\mathrm{G}}$ (where $R_{\mathrm{G}}=2634 \mathrm{~km}$ is the radius of Ganymede) measured from the moon's center into the direction of the incident flow. Our knowledge of Ganymede's magnetosphere and its internal magnetic field comes from the observations obtained by the Galileo spacecraft during its six close encounters with the moon (Kivelson et al. 1996, 1998). However, it was suggested by Kivelson et al. (2002) that only three of the six passes contain information relevant for determination of the internal field through quadrupole order. This was because two passes did not approach closely enough to detect higher order moments and one pass remained close to the boundary of Ganymede's magnetosphere where perturbations from large localized currents dominated the signal.

The magnetic field measured in the vicinity of Ganymede consists of several parts: Jupiter's ambient magnetospheric field, Ganymede's internal field and Ganymede's magnetospheric field arising from plasma interactions. The separation of external and internal field contributions was performed by using the data acquired in regions inside of Ganymede's magnetosphere that are relatively far from the magnetopause boundary where strong magnetospheric currents are present (Kivelson et al. 2002). The approach taken by Kivelson et al. (2002) was based on the classical spherical harmonic expansion described in Sect. 2 and based on the assumption that the data used for the analysis were collected in regions free of electric currents. Evident problems with this approach are that the spacecraft trajectory was not current-free and that it remained above the ionosphere, so ionospheric sources were combined with internal sources. The external contributions were solved only for the first-order $(n=1)$ terms of a spherical harmonic expansion, which corresponds to a uniform external field, but were allowed to vary from pass to pass. The internal contributions were 
solved for a set of fixed internal moments through quadrupole $(n=2)$ order and also for a combination of a fixed dipole and an induced dipole, whose orientation depends upon the instantaneous external driving field. The analysis imposed the obvious requirement that internal moments be identical on all passes. Both models provided equally satisfactory fits to the magnetic field data on multiple passes. The two different characterizations of the internal field could not be unambiguously distinguished based on the accuracy with which they reproduced the available flyby data. However, the model of permanent and induced dipole moments was favored because it requires fewer free parameters than does the model of a permanent dipole moment and quadrupole moments (Kivelson et al. 2002).

Uncertainties in the separation of external and internal contributions arise in part from the assumption that the external fields resulting from Ganymede's magnetospheric currents are spatially uniform. A more realistic representation of the magnetospheric fields can be provided by a physically motivated model of the global magnetosphere. Three-dimensional global MHD simulations, including single-fluid resistive MHD simulations (Kopp and Ip 2002; Ip and Kopp 2002; Jia et al. 2008) and multi-fluid MHD simulations (Paty and Winglee 2004, 2006; Paty et al. 2008), have been carried out to understand Ganymede's plasma and field environment. Among these, Jia et al. (2008) developed an MHD model that includes the moon's interior in the simulation domain and, therefore, has the advantage of including the magnetic diffusion effects in the moon's mantle. Moreover, their model used boundary conditions that are consistent with the available observations and a high resolution grid that enabled them to resolve the magnetospheric boundaries. The simulation results, in general, agree well with the magnetic field observations on multiple passes. However, the simulated magnetosphere is slightly smaller than that inferred from the measurements. Subsequently, Jia et al. (2009b) improved their model by applying an ionospheric boundary condition more appropriate for Ganymede and found that the simulated magnetosphere in the improved model is in extremely good agreement with the observations on multiple passes. The work therefore demonstrated that an MHD model can consistently describe the external plasma currents that contribute to the magnetic field measurements in the near-Ganymede environment. The new work also illustrated the sensitivity of global simulations to internal boundary conditions.

The external current system in Ganymede's magnetosphere identified from the MHD simulation is illustrated in Fig. 6. At low latitudes near Ganymede, there are two regions of intense currents flowing perpendicular to the magnetic field, one at the upstream magnetopause and the other in the downstream magnetotail (Fig. 6a). These are analogous to the classic Chapman-Ferraro magnetopause currents and the tail currents found in the terrestrial magnetosphere. Present at high latitudes are field-aligned currents (Fig. 6b), also called the Alfvén wing currents, which are generated mainly by the strong flow shear between the connected and external field lines. This current system is similar to the Region-1 current system in the Earth's magnetosphere. The currents flow towards Ganymede on the side closer to Jupiter and away from the moon on the side away from the planet. As shown by the current streamlines in Fig. 6c, the Alfvén wing currents close not only through the magnetopause and tail current sheets, but also through the moon and its ionosphere.

Although Ganymede's magnetosphere shares many properties with the planetary magnetospheres, there are noticeable differences in the global configuration resulting from different properties of the ambient plasma flow. In the super-Alfvénic solar wind, planetary magnetospheres form bullet-shape cavities in which the two tail lobes, normally containing open field lines, are roughly aligned with the direction of the incident solar wind. In contrast, in the sub-Alfvénic plasma flow typical at Ganymede's orbit, the magnetosphere forms into a roughly cylindrical shape with two nearly vertically extended Alfvén wings containing open field lines (see field lines in Fig. 6a). As a result, the Alfvén wing currents which 

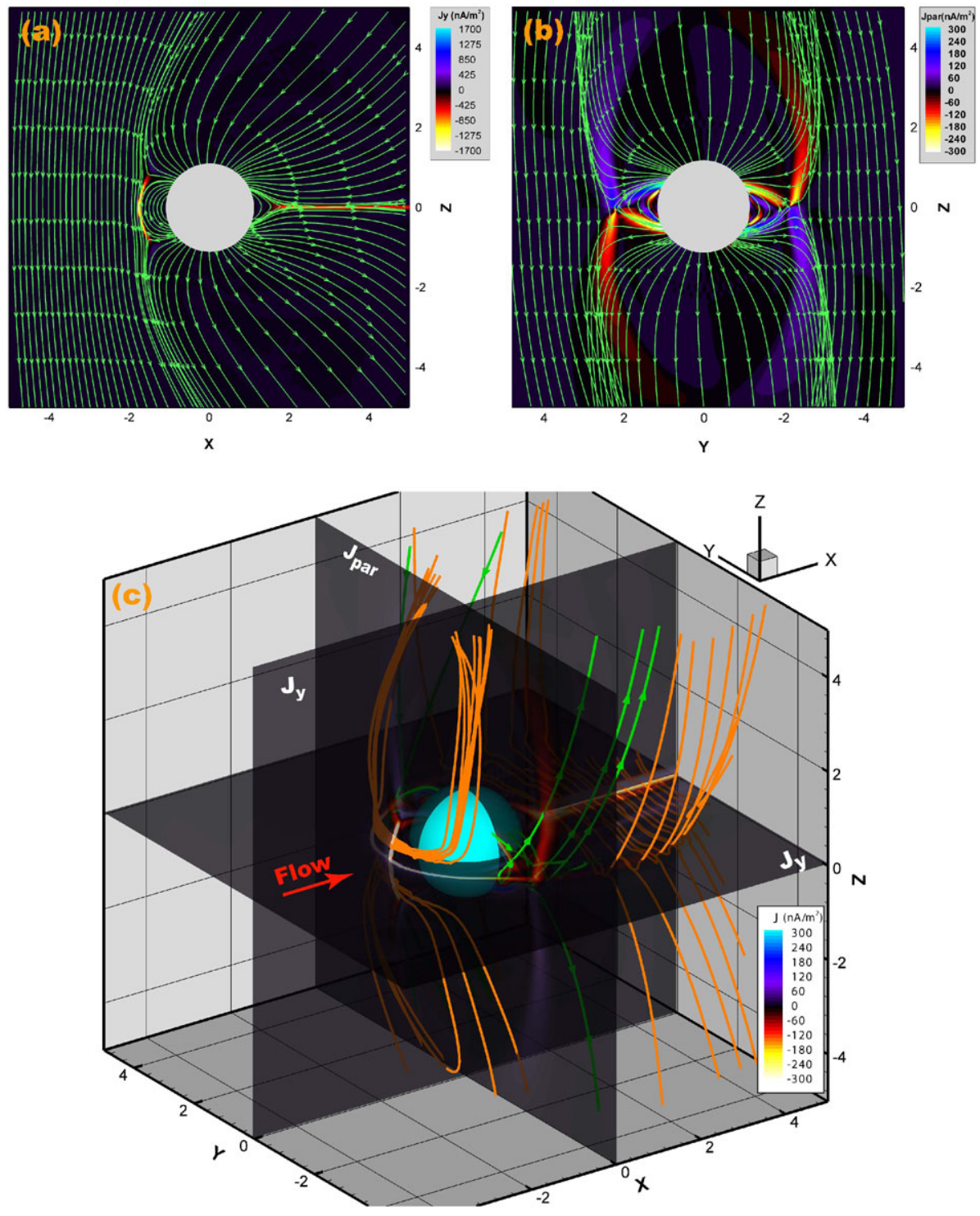

Fig. 6 (a) Color contours of the current density $\left(J_{y}\right)$ in the $\hat{Y}$ direction plotted in the $X Z$ plane at $Y=0$. The projection of magnetic field lines in this plane is superposed as green lines with arrowheads representing the field orientation. Note that density of field lines is arbitrary. (b) As for (a) but for the field-aligned current density $\left(J_{\text {par }}\right)$ in the $Y Z$ plane. Flow is into the plane. (c) A global view of the current flowlines in the magnetosphere from the upstream flank side. Results are shown in a Ganymede-centered Cartesian coordinates ("GPhiO"), where $\hat{X}$ is along the incident flow direction, $\hat{Y}$ is along the Ganymede-Jupiter vector, positive towards Jupiter, and $\hat{Z}$ is parallel to Jupiter's spin axis. The axes are labelled in units of Ganymede's radius $R_{\mathrm{G}}$. The figure is reproduced from Jia et al. (2008) 
go through Ganymede and its magnetosphere close far from Ganymede. Therefore, directly scaling empirical models of the magnetospheric currents developed in the context of a planetary magnetosphere to Ganymede's magnetosphere is not appropriate. In particular, a model such as that of Stone and Armstrong (2001) in which the external currents are specified by modifying empirically a model of Earth's magnetosphere, with the magnetopause and tail currents included, produces a magnetosphere whose global configuration differs greatly from that obtained in the MHD simulations (Jia et al. 2008, 2009b). In the Stone and Armstrong (2001) model, the magnetospheric currents, which separate the unperturbed external field and the magnetospheric field lines, close near Ganymede (within several Ganymede radii) whereas open flux tubes at Ganymede appear to link to Jupiter's ionosphere. The latter assertion is based on the auroral footprints (Clarke et al. 2002) that couple directly to Ganymede.

The perturbation field arising from external currents can be extracted directly from the MHD model. Figure 7 shows the magnetic field comparisons between the magnetometer measurements and the MHD simulations for two Galileo flybys, G2 and G28, that were included in the Kivelson et al. (2002) analysis. The G2 flyby is a polar pass with a closest approach altitude of $\sim 264 \mathrm{~km}\left(0.10 R_{\mathrm{G}}\right)$ and the $\mathrm{G} 28$ flyby is an upstream low-latitude pass with a closest approach altitude of $\sim 900 \mathrm{~km}\left(0.34 R_{\mathrm{G}}\right)$. Good agreement between the model results and the measurements allows us to identify the perturbation field quite accurately and thus to establish the degree of validity of the approximation in which the perturbation field is represented as a uniform field. The initial input in the MHD model consists of the background field (Jupiter's magnetospheric field) and Ganymede's internal field, including both the permanent and the induced dipole moments given by Kivelson et al. (2002). The difference between the simulated fields and the initial input observed along the spacecraft trajectory can then be considered as the perturbation field imposed by the external current system. For both flybys, the perturbation fields extracted from the MHD model deviate from uniform fields. In particular, for the G28 flyby, whose trajectory lies close to the upstream magnetopause, the perturbation field deviates considerably from a uniform field because the magnetopause shape and current density change significantly over small spatial scales. It should also be noted that the perturbation fields extracted from the comparison between the actual and the simulated data includes the contribution arising from the ionospheric currents, thereby avoiding a problem inherent in the spherical harmonic expansion of combining the contributions of ionospheric sources with sources internal to the moon.

The fact that the MHD simulation, which used the internal field model of Kivelson et al. (2002) as initial input, yields a rather good agreement with the observations implies that the dominant component of the internal field basically is reasonably well captured by the present internal field model. However, use of a good MHD model to extract plasma-driven effects should improve the determination of internal moments and the inductive response efficiency. Nonetheless, several factors need to be considered in such an approach. Firstly, results of MHD simulations depend largely on the boundary conditions at both the upstream and the inner boundary near the obstacle. In the planetary case, such as for Mercury, the upstream solar wind condition is highly variable and an accurate description of the magnetospheric currents requires knowledge of the instantaneous solar wind conditions. Fortunately in Ganymede's case, the upstream Jupiter's field and plasma conditions are relatively steady and predictable. However, properties of Ganymede's ionosphere, such as the conductivity, are poorly constrained observationally. Therefore, model results must be validated by comparing with spacecraft observations before being used to interpret the observations. Secondly, during the course of spacecraft measurements, the magnetosphere and the magnetospheric currents may exhibit temporal variations on time scales shorter than the time 
(a) G2 flyby in GphiO coordinates

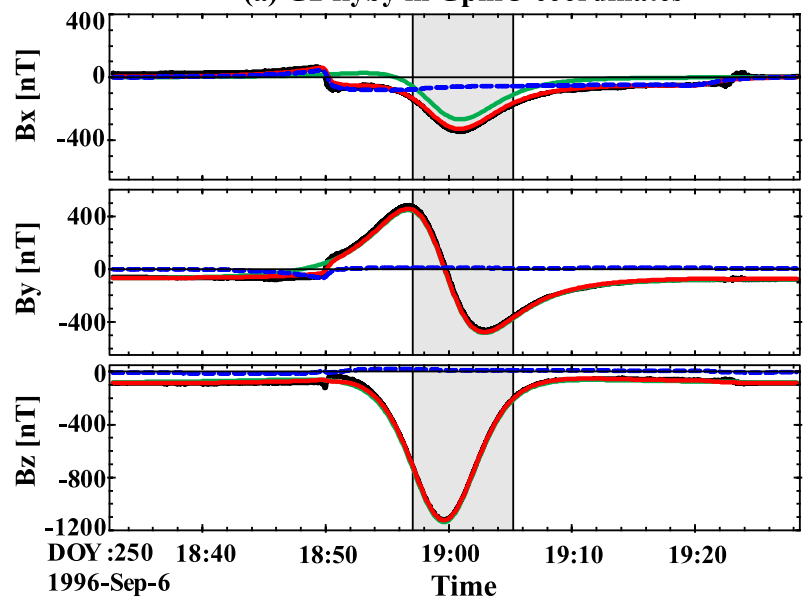

(c) G28 flyby in GphiO coordinates

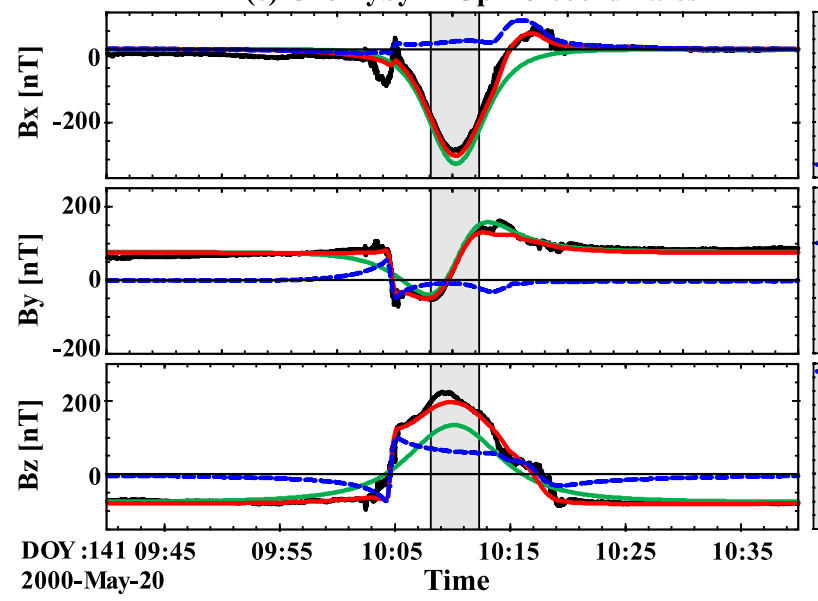

(b) Fitting interval

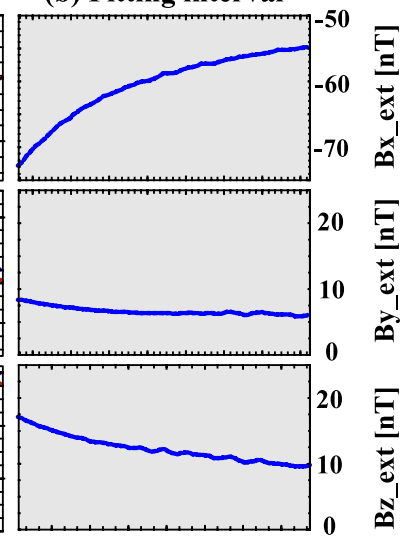

(d) Fitting interval

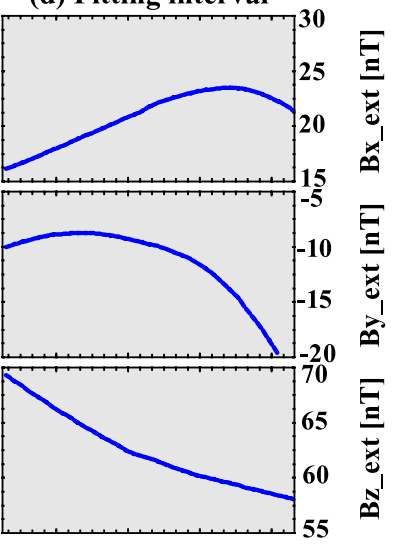

Fig. 7 Magnetic field comparisons between the Galileo measurements and MHD simulations for two Galileo flybys, G2 (a and b) and G28 (c and d). In panel (a) and (c), black solid lines are the Galileo data, green lines are the initial input in the MHD model and red traces are the simulation results. In order to account for the rapid temporal variations of Ganymede's magnetosphere resulting from reconnection on the magnetopause, simulation results shown in the comparisons are averaged over time steps that span a time interval of $\sim 2$ minutes after the system stabilizes. Blue dashed lines are the differences between red and green traces and they represent the perturbation fields due to the external current system produced in the MHD model. Grey shaded regions are the time intervals used by Kivelson et al. (2002) for the separation of internal-external fields. An expanded view of the external fields during the marked intervals is also shown in panel (b) and (d). Magnetic field vectors are shown in a Ganymede-centered Cartesian coordinates (GPhiO) defined in Fig. 6

interval of data collection owing to changes in the external plasma and field conditions or to dynamical processes in the magnetosphere (such as reconnection) that may be unsteady even under relatively steady external conditions. Under such circumstances, a steady state solution does not fully represent the variable external perturbations. Space-time aliasing of observations must be considered when the perturbation fields are inferred from simulations.

Another major limiting factor in fully characterizing Ganymede's internal field is insufficient data coverage. The magnetic field measurements at Ganymede were acquired during 
a limited number of flybys, which provided only a sparse coverage of the whole magnetosphere. The southern hemisphere, especially on the downstream side, has not been fully explored yet (see Fig. 9 in Jia et al. 2009a) and any magnetic anomaly localized in this region would have been "invisible". Even if the external plasma currents were fully known, the sparsity of data would remain a source of uncertainty. A complete determination of Ganymede's internal field requires more magnetic field measurements from future missions, especially in the unexplored high latitude regions in the southern hemisphere.

An optimal situation for accurately determining the internal field can be achieved by a polar orbiting spacecraft at altitudes below the current flowing region in the ionosphere. However, because the nature of Ganymede's ionosphere and atmosphere is not well constrained, it remains unclear where such a current-free region is located or even if it is present at all in Ganymede's environment. Until data from such an orbiter can be obtained, global models of the magnetospheric environment undoubtedly will play an essential role in characterizing the internal field and the inductive response of the moon.

\section{Conclusions}

Separating magnetic fields of internal and external origin is essential to infer details on the internally generated fields. The classical Gauss algorithm turns out to be very successful for studying the geomagnetic field, mainly because the field is dominated by a Laplacian potential field in the region where the measurements are taken. However, at other planetary bodies such as Mercury and Ganymede more elaborate techniques must be used before any firm conclusions can be drawn on the magnetic properties of these bodies. Measuring the magnetic field in a planetary environment is essential but not sufficient, and there is urgent need for further development of advanced analysis schemes. Field separation as discussed in this publication is an integral part of the interpretation process.

Acknowledgements NO highly appreciates valuable discussions with Michael E. Purucker on Mars data processing and model interpretation. XJ would like to thank Margaret Kivelson, Raymond Walker and Krishan Khurana for useful discussions. His work has been supported by NASA grant NNG05GB82G. The work by KHG was financially supported by the German Ministerium für Wirtschaft und Technologie and the German Zentrum für Luft- und Raumfahrt under contract 50 QW 0602.

Open Access This article is distributed under the terms of the Creative Commons Attribution Noncommercial License which permits any noncommercial use, distribution, and reproduction in any medium, provided the original author(s) and source are credited.

\section{References}

I.I. Alexeev, E.S. Belenkaya, S.Yu. Bobrovnikov, J.A. Slavin, M. Sarantos, Paraboloid model of Mercury's magnetosphere. J. Geophys. Res. 113(A12), 12210 (2008)

B.J. Anderson, M.H. Acuña, D.A. Lohr, J. Scheifele, A. Raval, H. Korth, J.A. Slavin, The magnetometer instrument on MESSENGER. Space Sci. Rev. 131, 417-450 (2007). doi:10.1007/s11214-007-9246-7

B.J. Anderson, M.H. Acuña, H. Korth, M.E. Purucker, C.L. Johnson, J.A. Slavin, S.C. Solomon, R.L. McNutt, The structure of Mercury's magnetic field from MESSENGER's first flyby. Science 321, 82 (2008). doi:10.1126/science.1159081

G. Backus, Poloidal and toroidal fields in geomagnetic field modeling. Rev. Geophys. 24, 75-109 (1986)

L. Bauer, Chief results of a preliminary analysis of the Earth's magnetic field for 1922. Terr. Magn. Atmos. Electr. 28(1), 1-28 (1923) 
W. Baumjohann, A. Matsuoka, W. Magnes, K.H. Glassmeier, R. Nakamura, H. Biernat, M. Delva, K. Schwingenschuh, T. Zhang, H.H.U. Auster, K. Fornaçon, U. Motschmann, I. Richter, A. Balogh, C. Carr, P.J. Cargill, M. Dougherty, T.S. Horbury, E.A. Lucek, T. Takahashi, M. Tanaka, T. Nagai, H. Tsunakawa, M. Matsushima, M. Shinohara, H. Kawano, A. Yoshikawa, H. Shibuya, T. Nakagawa, M. Hoshino, Y. Tanaka, R. Kataoka, B. Anderson, C. Russell, Magnetic field investigation of Mercury's magnetosphere and inner heliosphere environment by MMO/MGF. Planet. Space Sci. 57, (2009)

J.C. Cain, B.B. Ferguson, D. Mozzoni, An $n=90$ internal potential function of the Martian crustal magnetic field. J. Geophys. Res. (Planets) 108, 2-1 (2003). doi:10.1029/2000JE001487

S. Chapman, J. Bartels, Geomagnetism, vols. I+II (Clarendon Press, Oxford, 1940)

J.Y. Choe, D.B. Beard, The compressed geomagnetic field as a function of dipole tilt. Planet. Space Sci. 22, 595-608 (1974a)

J.Y. Choe, D.B. Beard, The near Earth magnetic field of the magnetotail current. Planet. Space Sci. 22, 609615 (1974b). doi:10.1016/0032-0633(74)90094-4

J.T. Clarke, J. Ajello, G. Ballester, L.B. Jaffel, J. Connerney, J.C. Gerard, G.R. Gladstone, D. Grodent, W. Pryor, J. Trauger, J.H. Waite Jr., Ultraviolet emissions from the magnetic footprints of Io, Ganymede and Europa on Jupiter. Nature 415, 997-1000 (2002)

M.W. Dunlop, A. Balogh, K.H. Glassmeier, P. Robert, Four-point cluster application of magnetic field analysis tools: The curlometer. J. Geophys. Res. 107, 23-12314 (2002). doi:10.1029/2001JA005088

E. Friis-Christensen, H. Lühr, G. Hulot, Swarm: A constellation to study the Earth's magnetic field. Earth Planets Space 58, 351-358 (2006)

H. Fritsche, Die Elemente des Erdmagnetismus und ihre säkularen Änderungen während des Zeitraumes 1550 bis 1900 (St. Petersburg, 1900)

C.F. Gauss, Allgemeine Theorie des Erdmagnetismus. Resultate aus den Beobachtungen des Magnetischen Vereins im Jahre 1838 (Göttinger Magnetischer Verein, Leipzig, 1839), pp. 1-52

G. Giampieri, A. Balogh, Modelling of magnetic field measurements at Mercury. Planet. Space Sci. 49, 1637-1642 (2001)

K.H. Glassmeier, H. Auster, D. Heyner, K. Okrafka, C. Carr, G. Berghofer, B.J. Anderson, A. Balogh, W. Baumjohann, P.J. Cargill, U. Christensen, M. Delva, M. Dougherty, K. Fornaçon, T.S. Horbury, E.A. Lucek, W. Magnes, M. Mandea, A. Matsuoka, M. Matsushima, U. Motschmann, R. Nakamura, Y. Narita, I. Richter, K. Schwingenschuh, H. Shibuya, J.A. Slavin, C. Sotin, B. Stoll, H. Tsunakawa, S. Vennerstrom, J. Vogt, T. Zhang, The fluxgate magnetometer of the BepiColombo planetary orbiter. Planet. Space Sci. 57, (2009)

J. Grosser, K.H. Glassmeier, A. Stadelmann, Induced magnetic field effects at planet Mercury. Planet. Space Sci. 52, 1251-1260 (2004). doi:10.1016/j.pss.2004.08.005

D. Heyner, Magnetfeldmessungen am Merkur: Einfluss externer Stromsysteme. Diploma thesis, Techn. Univ. Braunschweig (2007)

W.H. Ip, A. Kopp, Resistive MHD simulations of Ganymede's magnetosphere: 2. Birkeland currents and particle energetics. J. Geophys. Res. 107(A12), 1491 (2002). doi:10.1029/2001JA005072

D.J. Jackson, D.B. Beard, The magnetic field of Mercury. J. Geophys. Res. 82, 2828-2836 (1977). doi:10.1029/JA082i019p02828

X. Jia, R.J. Walker, M.G. Kivelson, K.K. Khurana, J.A. Linker, Three-dimensional MHD simulations of Ganymede's magnetosphere. J. Geophys. Res. 113, 6212 (2008). doi:10.1029/2007JA012748

X. Jia, M.G. Kivelson, K.K. Khurana, R.J. Walker, Magnetic fields of the satellites of Jupiter and Saturn. Space Sci. Rev. (2009a, this issue). doi:10.1007/s11214-009-9507-8

X. Jia, R.J. Walker, M.G. Kivelson, K.K. Khurana, J.A. Linker, Properties of Ganymede's magnetosphere inferred from improved three-dimensional MHD simulations. J. Geophys. Res. (2009b) doi:10.1029/2009JA014375

M.G. Kivelson, C.T. Russell, Introduction to Space Physics (Cambridge University Press, Cambridge, 1995)

M.G. Kivelson, K.K. Khurana, M. Volwerk, The permanent and inductive magnetic moments of Ganymede. Icarus 157, 507-522 (2002)

M.G. Kivelson, K.K. Khurana, C.T. Russell, R.J. Walker, J. Warnecke, F.V. Coroniti, C. Polanskey, D.J. Southwood, G. Schubert, Discovery of Ganymede's magnetic field by the Galileo spacecraft. Nature 384, 537-541 (1996)

M.G. Kivelson, J. Warnecke, L. Bennett, S. Joy, K.K. Khurana, J.A. Linker, C.T. Russell, R.J. Walker, C. Polanskey, Ganymede's magnetosphere: Magnetometer overview. J. Geophys. Res. 103, 1996319972 (1998)

A. Kopp, W.H. Ip, Resistive MHD simulations of Ganymede's magnetosphere: 1. Time variabilities of the magnetic field topology. J. Geophys. Res. 107(A12), 1490 (2002). doi:10.1029/2001JA005071

H. Korth, B.J. Anderson, M.H. Acuña, J.A. Slavin, N.A. Tsyganenko, S.C. Solomon, R.L. McNutt, Determination of the properties of Mercury's magnetic field by the MESSENGER mission. Planet. Space Sci. 52, 733-746 (2004). doi:10.1016/j.pss.2003.12.008 
R.A. Langel, The main field, in Geomagnetism, vol. 1, ed. by J.A. Jacobs (Academic Press, London, 1987), pp. 249-512

R.A. Langel, R.H. Estes, Large-scale, near-Earth magnetic fields from external sources and the corresponding induced internal field. J. Geophys. Res. 90, 2487-2494 (1985a)

R.A. Langel, R.H. Estes, The near-Earth magnetic field at 1980 determined from MAGSAT data. J. Geophys. Res. 90, 2495-2509 (1985b)

F.J. Lowes, Mean-square values on sphere of spherical harmonic vector fields. J. Geophys. Res. 71,2179 (1966)

H. Lühr, M. Korte, M. Mandea, The recent magnetic field and its variations, in Geomagnetic Variations, ed. by K. Glassmeier, H. Soffel, J.W. Negendank (Springer, Berlin, 2009), pp. 25-63

P. Mauersberger, Das Mittel der Energiedichte des geomagnetischen Hauptfeldes an der Erdoberfläche und seine säkulare Änderung. Gerl. Beitr. Geophys. 65, 207-215 (1956)

S. Maus, H. Lühr, Signature of the quiet-time magnetospheric magnetic field and its electromagnetic induction in the rotating Earth. Geophys. J. Int. 162, 755-763 (2005)

S. Maus, H. Lühr, G. Balasis, M. Rother, M. Mandea, Introducing POMME, the Potsdam magnetic model of the Earth, in Earth Observation with CHAMP, Results from Three Years in Orbit, ed. by C. Reigber, H. Lühr, P. Schwintzer, J. Wickert (Springer, Berlin, 2005), pp. 293-298

N.F. Ness, K.W. Behannon, R.P. Lepping, Y.C. Whang, K.H. Schatten, Magnetic field observations near Mercury: Preliminary results from Mariner 10. Science 185, 131-135 (1974)

N. Olsen, H. Lühr, T.J. Sabaka, M. Mandea, M. Rother, L. Tøffner-Clausen, S. Choi, CHAOS-a model of Earth's magnetic field derived from CHAMP, Ørsted, and SAC-C magnetic satellite data. Geophys. J. Int. 166, 67-75 (2006). doi:10.1111/j.1365-246X.2006.02959.x

N. Olsen, Ionospheric $F$ region currents at middle and low latitudes estimated from Magsat data. J. Geophys. Res. 102(A3), 4563-4576 (1997)

C. Paty, R. Winglee, Multi-fluid MHD simulations of Ganymede's magnetosphere. Geophys. Res. Lett. 31 (2004). doi:10.1029/2004GL021220

C. Paty, R. Winglee, The role of ion cyclotron motion at Ganymede: magnetic morphology and magnetospheric dynamics. Geophys. Res. Lett. 33 (2006). doi:10.1029/2005GL025273

C. Paty, W. Paterson, R. Winglee, Ion energization in Ganymede's magnetosphere: Using multifluid simulations to interpret ion energy spectrograms. J. Geophys. Res. 113 (2008). doi:10.1029/2007JA012848

C.T. Russell, R.J. Walker, Flux transfer events at Mercury. J. Geophys. Res. 90, 11067-11072 (1985)

T.J. Sabaka, N. Olsen, M. Purucker, Extending comprehensive models of the Earth's magnetic field with Ørsted and CHAMP data. Geophys. J. Int. 159, 521-547 (2004). doi:10.1111/j.1365-246X. 2004.02421.x

T.J. Sabaka, N. Olsen, Enhancing comprehensive inversions using the Swarm constellation. Earth Planets Space 58, 371-395 (2006)

J. Saur, F.M. Neubauer, K.H. Glassmeier, Induced magnetic field in solar system bodies. Space Sci. Rev. (2009, this issue)

N. Schilling, F.M. Neubauer, J. Saur, Time-varying interaction of Europa with the Jovian magnetosphere: Constraints on the conductivity of Europa's subsurface ocean. Icarus 192(1), 41-55 (2007). doi:10.1016/j.icarus.2007.06.024

A. Schmidt, Der magnetische Zustand der Erde zur Epoche 1885.0. Archiv der deutschen Seewarte, 21-75 (1898)

A. Schmidt, Mitteilungen äber eine neue Berechnung des erdmagnetischen Potentials. Abh. K. Bayrisch. Akad. Wiss. 19(1), 1-66 (1895)

J. Scuffham, A. Balogh, A new model of Mercury's magnetospheric magnetic field. Adv. Space Res. 38, 616-626 (2006). doi:10.1016/j.asr.2005.08.052

G. Siscoe, L. Christopher, Variations in the solar wind stand-off distance at Mercury. Geophys. Res. Lett. 2, 158-160 (1975)

J.A. Slavin, M.H. Acuña, B.J. Anderson, D.N. Baker, M. Benna, G. Gloeckler, R.E. Gold, G.C. Ho, R.M. Killen, H. Korth, S.M. Krimigis, R.L. McNutt, L.R. Nittler, J.M. Raines, D. Schriver, S.C. Solomon, R.D. Starr, P. Trávníček, T.H. Zurbuchen, Mercury’s magnetosphere after MESSENGER's first flyby. Science 321, 85 (2008). doi:10.1126/science. 1159040

S.M. Stone, T.P. Armstrong, Three-dimensional magnetopause and tail current model of the magnetosphere of Ganymede. J. Geophys. Res. 106(A10), 21263-21275 (2001)

N.A. Tsyganenko, A magnetospheric magnetic field model with a warped tail current sheet. Planet. Space Sci. 37, 5-20 (1989). doi:10.1016/0032-0633(89)90066-4

N.A. Tsyganenko, Modeling the Earth's magnetospheric magnetic field confined within a realistic magnetopause. J. Geophys. Res. 100, 5599-5612 (1995). doi:10.1029/94JA03193

N.A. Tsyganenko, A model of the near magnetosphere with a dawn-dusk asymmetry 1. Mathematical structure. J. Geophys. Res. 107(A8), 12-1 (2002a) 
N.A. Tsyganenko, A model of the near magnetosphere with a dawn-dusk asymmetry 2. Parameterization and fitting to observations. J. Geophys. Res. 107(A8), 10-1 (2002b)

N.A. Tsyganenko, M.I. Sitnov, Magnetospheric configurations from a high-resolution data-based magnetic field model. J. Geophys. Res. 112(A11), 6225 (2007). doi:10.1029/2007JA012260

H. Uno, C.L. Johnson, B.J. Anderson, H. Korth, S.C. Solomon, Modeling Mercury's internal magnetic field with smooth inversions. Earth Planet. Sci. Lett. (2009) doi:10.1016/j.eps1.2009.02.032

Y.C. Whang, Magnetospheric magnetic field of Mercury. J. Geophys. Res. 82, 1024-1030 (1977). doi:10.1029/JA082i007p01024 